\title{
Contrast-enhanced ultrasonography of the rabbit VX2 tumor model: Analysis of vascular pathology
}

\author{
YANJUN LIU ${ }^{1}$, WEIDONG REN ${ }^{1,2}$, CAIGANG LIU ${ }^{3}$, KUN HUANG ${ }^{1}$, \\ YUEQIN FENG $^{1}$, XUEMEI WANG $^{1}$ and YUZHANG TONG ${ }^{4}$
}

\author{
${ }^{1}$ Department of Ultrasound, First Affiliated Hospital, China Medical University, Shenyang $110001 ;{ }^{2}$ Department of Ultrasound, \\ Shengjing Hospital, China Medical University, Shenyang 110004; ${ }^{3}$ Department of Breast Surgery, General Surgery, \\ The First Hospital of China Medical University; ${ }^{4}$ Department of Regional Anatomy, \\ China Medical University, Shenyang 110001, P.R. China
}

Received March 17, 2012; Accepted June 27, 2012

DOI: $10.3892 / \mathrm{ol} .2012 .819$

\begin{abstract}
The accuracy of diagnosing tumors may be improved significantly by detecting the microvascular distribution. Indeed, contrast-enhanced ultrasonography (CEUS) has shown a distinct advantage in detecting microvasculature. This study aimed to determine the angiogenic characteristics of VX2 tumors in rabbits using CEUS. A total of 17 rabbits were injected with $0.5 \mathrm{ml} \mathrm{VX2}$ cell suspension into the muscles of both hind legs to prepare the VX2 tumor models. At 14, 21, 28 and 35 days after tumor inoculation, CEUS was performed on the rabbits with $0.3 \mathrm{ml}$ SonoVue following a local anesthesia. The pathological findings of the tumors were compared. A total of 12 rabbits survived after being inoculated with the tumor cells and developed a total of 38 tumors. The size of the tumors ranged from 1.12 to $10.85 \mathrm{~cm}$. Using CEUS, all tumors demonstrated rim enhancement with some unenhanced regions. Enhancement began from the peripheral region and quickly showed internal reticular vessels. Regardless of the tumor size or the presence of necrosis, no complete enhancement of the tumors was observed. On microscopic examination, VX2 tumor cells were detected in striated muscles, immature blood capillaries and fibrosis tissues scattered in tumor nests. Immunohistochemical examination revealed that $\mathrm{CD} 34^{+}$cells appeared mainly in the muscles adjacent to vessels. In conclusion, CEUS may be an efficient method to evaluate angiogenesis and blood perfusion in VX2 tumors.
\end{abstract}

Correspondence to: Professor Weidong Ren, Department of Ultrasound, First Affiliated Hospital, China Medical University, No. 36 Sanhao Street, Heping, Shenyang 110001, P.R. China E-mail: zouxiaohui2000@163.com

Key words: contrast-enhanced ultrasonography, VX2 tumor, rabbit, animal model

\section{Introduction}

The VX2 tumor cell line originated from Shope virus-induced human papilloma-derived squamous cell carcinoma and was established after 72 transplantation passages (1). The VX2 tumor cell line may be used to establish animal tumor models. The rabbit VX2 tumor is induced in the skin by Shope virus and is characterized by easy inoculation, rapid growth, and significant invasiveness. These factors are similar to the characteristics of human squamous cell carcinoma (2).

SonoVue, a novel ultrasound reagent, forms microbubbles after resolution (3). It contains sulfur hexafluoride and is coated with a stable phospholipid sheath. The diameter of SonoVue microbubbles is 5 to $7 \mu \mathrm{m}$, similar to the size of normal human red blood cells. This enables them to enter microvessels, including new tumor vessels (4). Contrast-enhanced ultrasonography (CEUS) was developed on the basis of the pharmaceutical and ultrasonic characteristics of SonoVue microbubbles and may improve the ultrasonic exhibition of microvasculature.

In the current study, we performed CEUS on VX2 tumors and used a CD34 antibody (a vascular endothelial marker) to label tumor microvessel endothelial cells. We then compared the ultrasonic results of tumor microvessel perfusion and pathological results of tumor angiogenesis. The goal was to investigate the value of CEUS in diagnosing soft tissue tumors and determine the correlation between tumor microvessel perfusion and distribution.

\section{Materials and methods}

Animals, main instruments and agents. A total of 17 healthy New Zealand white rabbits, aged from two to three months and weighing from 2.0 to $2.8 \mathrm{~kg}$, were obtained from the Experimental Animal Department, China Medical University (Shenyang, China). The Medical Imaging Institute of China Medical University provided VX2 tumor tissues, stored at $-198^{\circ} \mathrm{C}$. All procedures were conducted with the approval of the Animal Research Committee at China Medical University.

An Acuson Siemens Sequoia-512 color Doppler sonographic system (Siemens, Erlangen, Germany), equipped with 
coherent pulse sequence (CPS) settings for small organs and 15L8Ws transducers (frequency range of 7-12 MHz) were used for conventional and contrast ultrasound scans. A HHW21.600 isothermal hot water cylinder (Hangzhou Aipu Equipment Co. Ltd., Zhejiang, China) was used to thaw the VX2 tumor tissue.

The ultrasound contrast agent SonoVue (Braco, Milan, Italy) was used for the contrast ultrasound scans. A Sumianxin injection $(1 \mathrm{ml} / \mathrm{ampul})$, produced by the military Veterinary Institute of the Academy of Military Medical Sciences and composed of amine thiazole xylene, ethyl diamine tetraacetic acid, hydrochloric acid and dihydroetorphine was used to anesthetize the rabbits. CD34 monoclonal antibody was used for S-P immunohistochemical staining.

Preparing the VX2 tumor models in rabbits. The VX2 tumor tissues were thawed at 36 to $37^{\circ} \mathrm{C}$ for $15 \mathrm{~min}$. They were then placed in a sterile plate containing normal saline and were cut into $1-\mathrm{mm}^{3}$ pieces to prepare the tumor tissue suspension. One rabbit was injected with a Sumianxin injection $(0.1 \mathrm{ml} / \mathrm{kg}$ body weight) for local anesthesia, then injected with $0.5 \mathrm{ml}$ of VX2 tumor tissue suspension in the vastus medialis muscle of both hind legs. This procedure prepared the animal model of the VX2 tumor. The developed VX2 tumor was removed surgically 21 days after inoculation. The peripheral tumor tissue, which had grown rapidly, was cut into $\sim 1-\mathrm{mm}^{3}$ pieces and suspended in $5 \mathrm{ml}$ normal saline. The tumor tissue suspension was then injected into the vastus medialis muscle of both hind legs $(0.5 \mathrm{ml}$ for each side) of the remaining 16 rabbits after injecting local anesthesia.

Conventional and contrast-enhanced ultrasonography. Ultrasonography was performed on days 14, 21, 28, and 35 after tumor inoculation. At each time point, three rabbits were fixed on an experiment table in the supine position following intramuscular anesthesia with Sumianxin injection $(0.1 \mathrm{ml} / \mathrm{kg}$ body weight). Rabbit fur at the hind legs, inguinal regions and lower abdomen were removed for ultrasonic observation.

Conventional ultrasonography was performed to observe the tumors and select the maximum tumor section for contrast ultrasonic observation. The SonoVue injection was resolved in normal saline at a ratio of $1: 5 \mathrm{ml}$ and agitated for $20 \mathrm{sec}$ for complete dissolution. Each rabbit underwent bolus injection of $0.3 \mathrm{ml}$ of SonoVue solution and a quick injection of $5 \mathrm{ml}$ of normal saline via the ear margin vein through a three-way tube. Coherent pulse sequence and tissue equalization techniques were performed with a low mechanical index of 0.19 , a frame rate of 200 frames/sec and a trigger time $(\Delta \mathrm{T})$ of $125 \mathrm{msec}$. Dynamic images following SonoVue injection were recorded until the contrast agent diminished.

Image processing and data analysis. Dynamic images were reviewed by two ultrasound physicians to record the enhancement patterns of the tumors. Enhanced areas at the margin and inside the VX2 tumors were set as regions of interest (ROIs) by ACQ software to develop time-intensity curves of all ROIs and measure arrival time (AT), time to peak (TTP) and peak intensity (PI) for different areas of the tumor. Adjacent vessels were avoided during measurement. Three intratumoral and three marginal ROIs were selected to calculate average values.
Histopathological examination. Following CEUS, the rabbits were euthanized using air embolization. VX2 tumors were excised, fixed in a neutral formalin solution for $24 \mathrm{~h}$ and embedded in paraffin. Tumor sections were prepared for hematoxylin-eosin (HE) staining and S-P immunohistochemical staining with CD34 monoclonal antibody to label the tumor microvessels. HE-stained sections and CD34-stained microvessels (in brown) were observed under a Nikon light microscope (Japan) by a pathologist. The correlations between the histopathological findings of the tumor morphology and the microvessel distribution and CEUS findings were analyzed.

\section{Results}

VX2 tumor formation in rabbits. Of the 16 rabbits, four died at $1,3,6$ and 8 days after tumor inoculation. A total of 38 tumors developed in the remaining 12 rabbits; nine had multiple lesions, whereas three had a single lesion. The tumors grew quickly at 14 days after inoculation. The size of the tumors ranged from $1.12 \times 1.35 \mathrm{~cm}$ to $10.85 \times 7.80 \mathrm{~cm}$.

Conventional ultrasonic findings of VX2 tumors. Along with tumor growth, the internal echoes of tumors on two-dimensional ultrasonography changed gradually. At 14 days after tumor inoculation, homogeneous hypoechoes were observed in the tumors (Fig. 1A). At 21 days after tumor inoculation, internal echoes were heterogeneous, changing from scattered dotted hyperechoes to central dotted hypoechoes (Fig. 1B). At 35 days after tumor inoculation, residual separate echoes were observed, but only in the remaining cysts. The diameter of the minimal tumor with echo changes was only $2 \mathrm{~cm}$. Color Doppler flow imaging and color Doppler energy imaging revealed peripheral distribution of irregular blood branches in VX2 tumors (Fig. 1C).

Contrast-enhanced ultrasonography. CEUS showed marked peripheral enhancement of the VX2 tumors at the early arterial phase and transient increased enhancement, then quick washout of contrast agents. All tumors showed rim enhancement at $\sim 6 \mathrm{sec}$ after the contrast agent was injected (Fig. 2A) and a little intratumoral enhancement at 12 to $18 \mathrm{sec}$ (Fig. 2B and C). Tumor contours were clear as the surrounding muscles showed no enhancement. The central areas of the tumors with hypoechoes on conventional ultrasonography showed irregular weak enhancement, whereas the anechoic necrotic areas showed no enhancement.

CEUS time-intensity curves of VX2 tumors showed a subdued uplift with a blunt peak, indicating slow enhancement, and a subdued decrease lasting $~ 40 \mathrm{sec}$, on average, indicating slow washout of the contrast agent (Fig. 3). As analyzed by the ACQ software, BI (base intensity) was $8.61 \pm 5.89 \mathrm{db}$, A (slope of the ascending curve) was $9.7 \pm 0.3 \mathrm{db}$, AT was $6.91 \pm 1.01 \mathrm{sec}$, TTP was $11.3 \pm 3.5 \mathrm{sec}$ and PI was $9.85 \pm 8.39 \mathrm{db}$.

Pathological findings. The surface of the tumors had a high tension, with vessel dilation and a pseudocapsule. The tumors were white and cystic with necrosis in the central area and unclear boundaries with the surrounding normal muscles (Fig. 4A). 

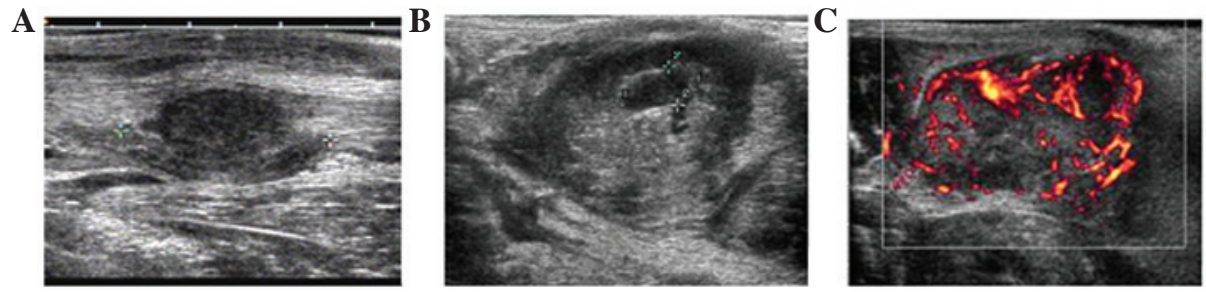

Figure 1. Conventional ultrasonography of VX2 tumors in rabbits. (A) At 14 days after tumor inoculation, 2-D ultrasonography showed homogeneous internal hypoechoes (calipers) in VX2 tumors with clear contour. (B) At 21 days after tumor inoculation, 2-D ultrasonography showed hypoechoes in tumors with clear contour and irregular anechoic areas (calipers) with stripped septate echoes. (C) At 21 days after tumor inoculation, color Doppler energy imaging showed blood flow signals in the peripheral regions and irregular blood branches inside the VX2 tumors.
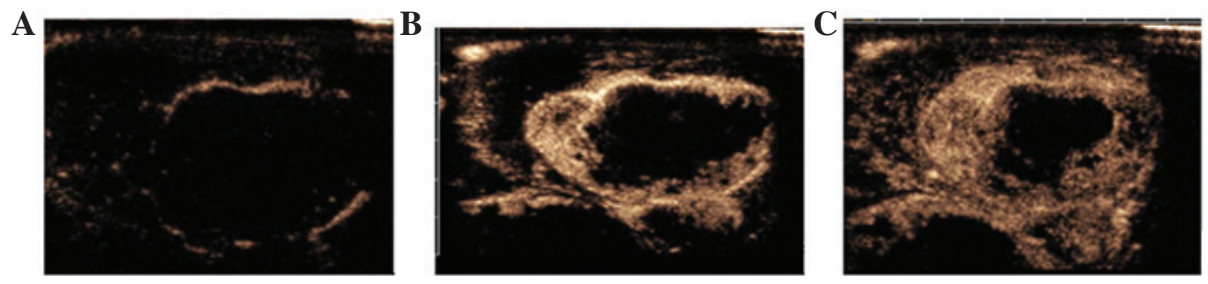

Figure 2. Contrast-enhanced ultrasonography (CEUS) of VX2 tumors in rabbits. (A) At 6 sec after contrast agent injection, CEUS showed rim enhancement surrounding the tumor. (B) At $12 \mathrm{sec}$ after contrast agent injection, CEUS showed a little intratumoral enhancement. (C) At 18 sec after contrast agent injection, CEUS showed increased enhancement inside the tumor, with the exception of the necrotic area.

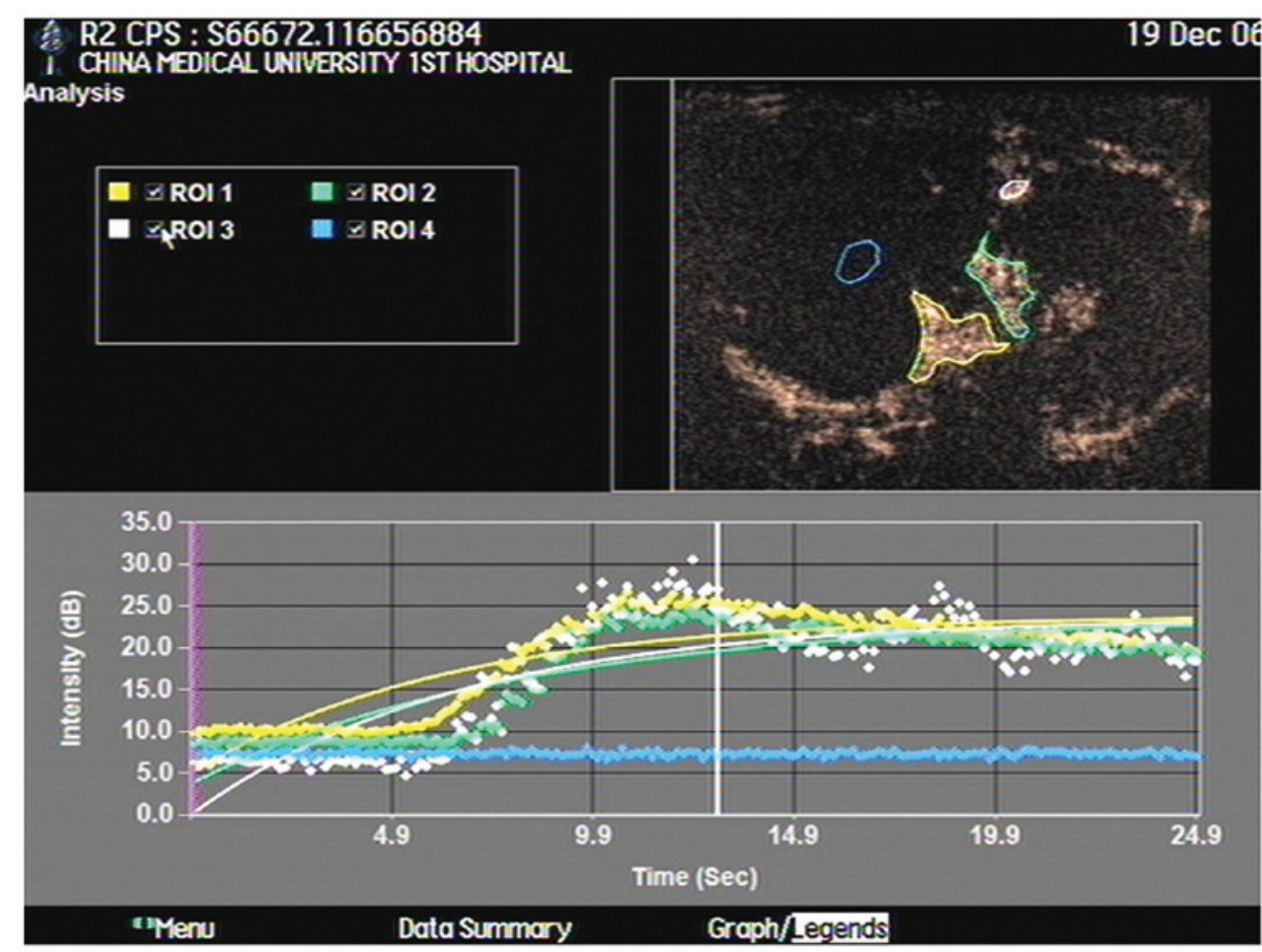

Figure 3. CEUS time-intensity curves of VX2 tumors analyzed by the ACQ software. The flat blue curve indicates the necrotic area with no enhancement. CEUS, contrast-enhanced ultrasonography.

On microscopic examination, the VX2 tumor cells that exhibited large nuclei with pathological mitosis were nested or scattered in striated muscles (Fig. 4B). Abundant immature capillaries and fibrosis tissues were distributed in the tumor nests (Fig. 4C). Degenerating tumor cells with no nuclei were observed in necrotic areas at 28 days after tumor inoculation. CD34-positive vascular endothelial cells (in brown) were detected in peripheral areas of the VX2 tumors and interstitial tissues (Fig. 4D and E).

Correlation between pathological and CEUS findings. The scope of the tumor enhancement on CEUS imaging was consistent with the distribution of CD34-positive vessels. All VX2 tumors showed the same enhancement pattern; that is, quick 
A

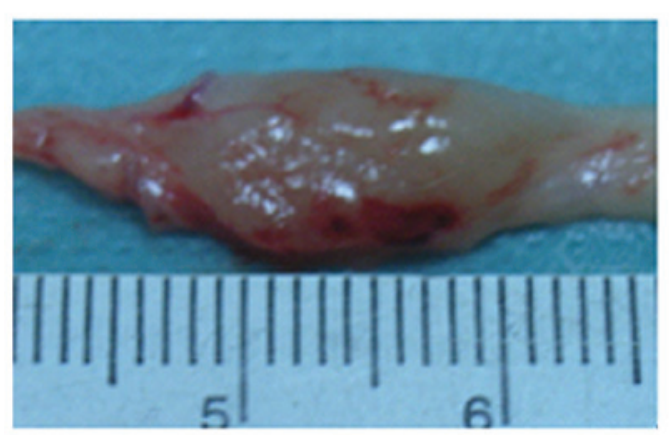

C

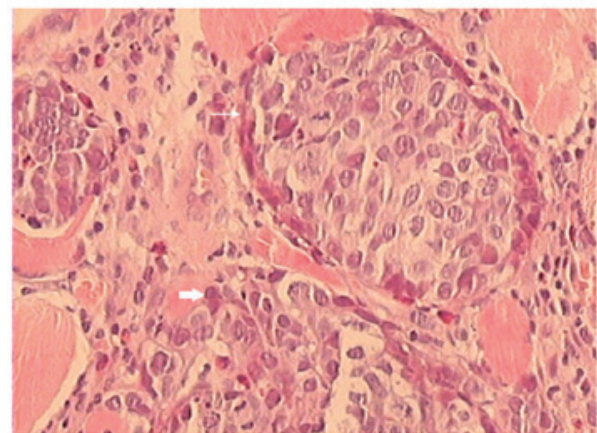

B

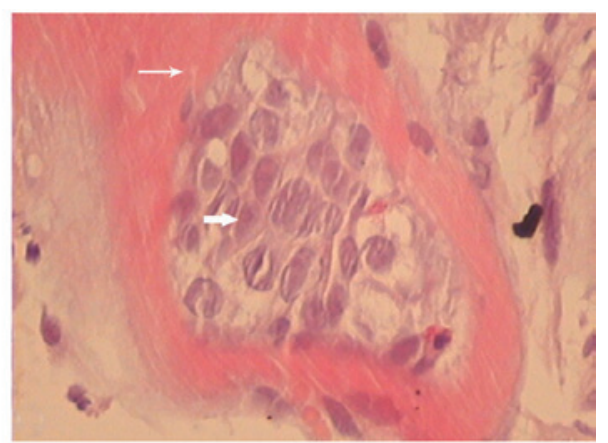

D

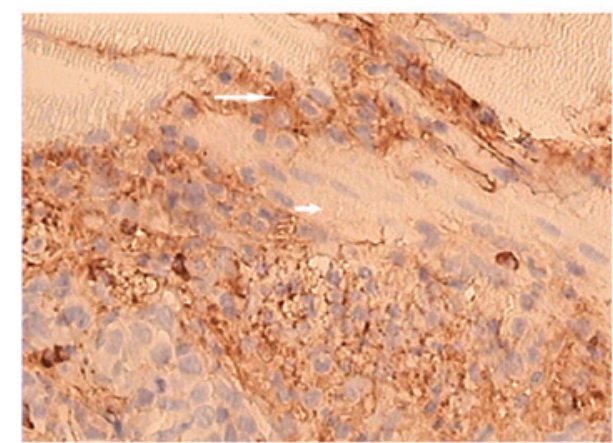

$\mathbf{E}$

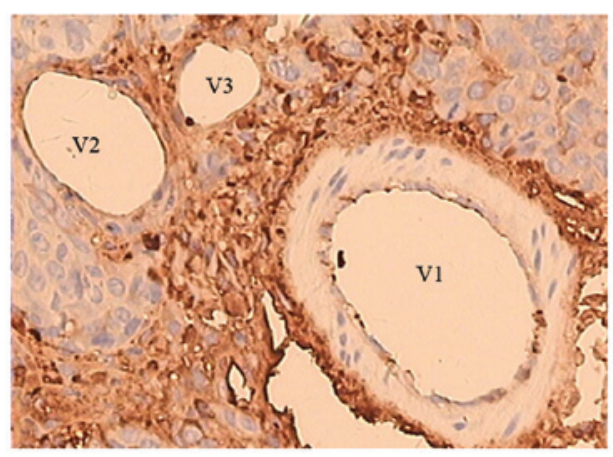

Figure 4. Histopathological examination of VX2 tumors in rabbits. (A) Gross specimen of the VX2 tumor shows pink tumor tissues with unclear boundary to normal muscle tissues. (B) VX2 tumor cells (short arrow) were scattered in the striated muscles (long arrow; HE, x200). (C) Fibrous capsules (long arrow) surrounded the tumor cell clusters; some tumor cells (short arrow) were scattered in the muscle tissue (HE, x100). (D) Brown CD34-positive regions (long arrow) were scattered or clustered at the peripheral areas of the VX2 tumor, but did not form a lumen; normal striated muscles (short arrow) were not stained (IHC, x100). (E) Brown CD34-positive regions adjacent to an arterial vessel with a thick wall (V1). Of the two venous vessels near the arterial vessel; the larger (V2) is completely surrounded by CD34-positive cells, while the smaller (V3) is incompletely surrounded (IHC, x100). HE, hematoxylin-eosin; IHC, immunohistochemical staining.

perfusion and transient enhancement at the arterial phase, with no capillary or venous phase. The enhancement first appeared in the peripheral regions of the tumors, then quickly showed in internal reticular vessels. No complete intratumoral enhancement was observed despite the tumor size and the presence of necrosis. Immunohistochemical staining revealed that the CD34-positive cells were scattered or clustered mainly in the muscles adjacent to vessels and peripheral tumor tissues, but could not form normal vessel lumens. No CD34-positive cells were observed in necrotic areas.

\section{Discussion}

The invasion and metastasis routes of VX2 tumors are similar to those ofhuman head and neck squamous cell and hepatocellular carcinomas $(5,6)$. Animal models of VX2 tumors, therefore, have commonly been used to study these cancers $(7,8)$. When planted to the bottom of culture bottles,
VX2 tumor cells grew in a single layer with partially overlapping areas. Multinuclear giant cells were also observed. Cells proliferated quickly with 58 to 62 hypotriploid chromosomes. The success rates of inoculation in both athymic nude mice and homogeneous rabbits were 100\% (9). VX2 tumors overexpress multiple matrix metalloproteins during the invasion process (10). The methods used, therefore, represent a good model of tumor metastasis and invasion.

In this study, we implanted VX2 tumors in the hind leg muscles of rabbits. Pathological examination confirmed the intramuscular distribution of tumor cells, indicating that VX2 tumor-bearing animal models were established successfully. Tumors grew quickly in the first three weeks and showed necrosis and cysts at advanced stages, which were consistent with VX2 tumor characteristics $(11,12)$. Due to the similarities between VX2 tumor and human carcinomas, determining the acoustic characteristics of VX2 tumors may contribute to the diagnosis of human tumors. 
Tumor growth relies on tumor feeding arteries. Forming tumor vessels is a complex process, which currently cannot be assessed directly or effectively. Tumor vessel endothelial cells may be labeled using immunohistochemistry. For example, the CD34 antibody is expressed in the cytoplasm of active vessel endothelial cells (13). Assessing the distribution and density of CD34-positive vessels aids the determination of tumor angiogenesis. Tumor specimens, however, cannot be used to evaluate the activity of new tumor vessels. In this study, we used CEUS, a non-invasive imaging method, to demonstrate tumor microvessel perfusion and assess tumor angiogenesis in vivo.

Using color Doppler flow and energy imaging to assess the distribution of tumor vessels is based on color and the energy signals of the tumor vessels. As it is limited by spatial resolution, it is prone to disturbance and cannot fully detect flows in microvessels. The lipid membrane structure, good in vivo stability of SonoVue, a second generation contrast agent, and the combination of acoustic features and second harmonic imaging improve the accuracy of CEUS. Contrast-enhanced gray-scale ultrasonography with a low mechanic index, a real-time imaging method with high spatial resolution, shows enhancements similar to that of computed tomography (CT) (14).

In this study, we assessed tumor vessel perfusion on CEUS and analyzed its correlation with tumor morphology and CD34-positive tumor vessel distribution. CEUS images of VX2 tumors demonstrated marked enhancements at the tumor margins. Pathological examination also revealed that CD34-positive regions increased and accumulated in the muscles close to normal vessels, suggesting that new tumor supply vessels are formed from the sites of existing vessels and provide nutrition to the tumor cells. Recent studies have found that tumor cells cannot live without sufficient oxygen and nutrition or protection from toxic molecules. Oxygen disseminates to capillaries from a distance of 150 to $200 \mu \mathrm{m}$ to tumor cells (15). To continue growing, tumor cells need continuous nutrition supplied by new vessels. New vessels are formed by either budding or non-budding. Budding develops new capillaries from existing vessels; in non-budding, tumor cells proliferate under the mediation of existing vascular endothelial cells, divide extensively and fuse to form new vessels.

Using CEUS, we found that all VX2 tumors exhibited the same enhancement pattern: quick perfusion and transient enhancement at the arterial phase, with no capillary or venous phase. Enhancement was first shown in the peripheral regions of tumors, then quickly showed in internal reticular vessels. No complete intratumoral enhancement was observed despite the tumor size and the presence of necrosis. Other researchers have transplanted VX2 tumor cells to the liver or kidneys in rabbits and also found tumor periphery-dominant enhancement on CEUS (16). We labeled tumor vessel endothelial cells by immunohistochemistry and found increased new vessels in the peripheral areas of tumors. Their basement membranes, however, were incomplete and did not form normal vessel lumens, presenting as scattered or clustered CD34-positive areas. In addition, these CD34-positive areas around arteries were more abundant than those found around veins, suggesting that the transient enhancement of VX2 tumors at arterial phase is related to new arteries formed in tumors.
Pathological examination confirmed the unenhanced tumor tissues as coagulation necrosis with cell apoptosis and revealed no CD34-positive new tumor vessels. This local necrosis differs from the liquefaction necrosis shown on conventional ultrasonography. Local necrosis presents homogenous solid hypoechoes, whereas liquefaction necrosis presents liquid or dotted echoes. This difference is more marked when assessing tumor vessel perfusion on CEUS. Pathological examination revealed that hemorrhagic necrotic regions existed in tumor tissues, but their acoustic impedance difference might not be large enough to be reflected using ultrasound. This minimal difference may be detected only on CEUS; therefore, the CEUS findings accurately reflect tumor vessel perfusion and indicate that new vessels in tumors are forming and distributing.

When analyzing the CEUS findings of solid tumors with a single blood supply using the ACQ software, we should pay attention to the length of the entire tumor enhancement phase (reflecting the distribution of arteries or veins and the maturity of vessels) and to the enhancement pattern and intensity of peripheral tumor vessels (relating to forming and distributing new vessels). For the tumor areas with weak or no enhancement, the CEUS findings should be analyzed, taking into account the pathological findings. Not all solid areas present homogenous enhancement; not all unenhanced areas are necrotic areas. The characteristics of the contrast agent determine whether CEUS reflects only the distribution of tumor microvessels. Clinical studies have shown that the distribution and quantity of tumor vessels determine the tumor enhancement pattern. Illustrating tumor blood supply aids the differentiation of benign and malignant solid tumors.

Whether the transplanted VX2 tumors mimic the angiogenesis of original or metastatic human tumors fully needs to be investigated. Room for improvement exists in selecting the maximal section, the influence of the contrast agent dose, time recording, the stability of operators and the selection of ROIs.

In conclusion, CEUS combined with administering the contrast agent SonoVue may be used to assess angiogenesis and blood perfusion in VX2 tumors in rabbits. Investigating tumor angiogenesis with CD34 immunohistochemical staining is the pathological basis for CEUS on VX2 tumors. The enhancement pattern of VX2 tumors is consistent with tumor vessel distribution.

\section{References}

1. Georges E, Breitburd F, Jibard N and Orth G: Two Shope papillomavirus-associated VX2 carcinoma cell lines with different levels of keratinocyte differentiation and transplantability. J Virol 55: 246-250, 1985.

2. Li C, Wang W, Ding H, Huang B, Cao J, Mao F and Ji Z: Value of contrast-enhanced sonography in the diagnosis of peripheral intrahepatic cholangiocarcinoma. J Clin Ultrasound 39: 447-453, 2011.

3. Domenech E, Berná-Serna Jde D, Polo L, Reus M, Berná-Mestre Jde D and Canteras M: Effect of SonoVue on the synovial membrane in rabbit knees. J Ultrasound Med 30: 1241-1246, 2011.

4. Tao S, Qin Z, Hao W, Yongquan L, Lanhui Y and Lei Y: Usefulness of gray-scale contrast-enhanced ultrasonography $\left(\right.$ SonoVue ${ }^{\circledR}$ ) in diagnosing hepatic alveolar echinococcosis. Ultrasound Med Biol 37: 1024-1028, 2011.

5. Lee KC, Moon WK, Chung JW, et al: Assessment of lymph node metastases by contrast-enhanced MR imaging in a head and neck cancer model. Korean J Radiol 8: 9-14, 2007. 
6. Sonoda A, Nitta N, Nitta-Seko A, et al: Time-course studies of implanted rabbit VX2 liver tumors to identify the appropriate time for starting hepatic arterial embolization in animal models. Oncology 80: 92-96, 2011.

7. Dünne AA, Mandic R, Ramaswamy A, et al: Lymphogenic metastatic spread of auricular VX2 carcinoma in New Zealand white rabbits. Anticancer Res 22: 3273-3279, 2002.

8. Dünne AA, Schmidt A, Kuropkat C, Ramaswamy A, Schulz S and Werner JA: The auricular VX2 carcinoma--an animal mode for sentinel node concept. In Vivo 17: 457-461, 2003.

9. Liu XF, Ren LR, Su GY, et al: Establishment and characterization of a rabbit tumor cell line VX2. Zhonghua Bing Li Xue Za Zhi 34: 661-663, 2005 (In Chinese).

10. Mandic R, Dünne AA, Eikelkamp N, et al: Expression of MMP-3, MMP-13, TMP-2 and TMP-3 in the VX2 carcinoma of the New Zealand white rabbit. Anticancer Res 22: 3281-3284, 2002.

11. Li J, Dong B, Yu X and Li C: Ultrasonographic portography with low mechanical index gray-scale imaging in hepatic VX2 tumor. Ultrasound Med Biol 32: 641-647, 2006.
12. Maruyama H, Matsutani S, Saisho H, Kamiyama N, Mine Y, Hirata $\mathrm{T}$ and Sasamata M: Sonographic shift of hypervascular liver tumor on blood pool harmonic images with definity: timerelated changes of contrast-enhanced appearance in rabbit VX2 tumor under extra-low acoustic power. Eur J Radiol 56: 60-65, 2005.

13. Simmons DL, Satterthwaite AB, Tenen DG and Seed B: Molecular cloning of a cDNA encoding CD34, a sialomucin of human hematopoietic stem cells. J Immunol 148: 267-271, 1992.

14. Xing N, Cai ZL, Zhao SH, Yang L, Xu BX and Wang FL: The use of CT perfusion to determine microvessel density in lung cancer: Comparison with FDG-PET and pathology. Chinese J Cancer Res 23: 118-122, 2011.

15. Fidler IJ and Ellis LM: Neoplastic angiogenesis - not all blood vessels are created equal. N Engl J Med 351: 215-216, 2004.

16. Elagoz S, Egilmez R, Koyuncu A, Muslehiddinoglu A and Arici S: The intratumoral microvessel density and expression of bFGF and $\mathrm{nm} 23-\mathrm{H} 1$ in colorectal cancer. Pathol Oncol Res 12: 21-27, 2006. 